\title{
Tratamiento quirúrgico de la presbicia con lentes intraoculares
}

\section{Revisión}

Torres-Bernal LF*, Rosas-Cabral A*, Terrones-Saldivar MC*, Valdepeña-López Velarde VD**.

-

- El advenimiento y desarrollo de nuevos lentes intrao- culares (LIO's) ha permitido que la cirugía de catara-

- ta evolucione a ser una cirugía refractiva, en donde

- los nuevos LIO's, remplazan de forma satisfactoria el

- cristalino natural, lo que representa uno de los pilares

- en el tratamiento de la presbicia. El presente artículo

- tiene como objetivo el de dar a conocer las caracterís-

- ticas de los nuevos LIO's para presbicia, los resultados

- clínicos obtenidos y los retos a los que se enfrenta

- el cirujano, que involucran la selección adecuada del

- paciente en base a sus expectativas y necesidades, la

- selección del lente intraocular adecuado, asi como la

- evaluación preoperatoria y los cuidados postoperato-

- rios necesarios para lograr la máxima satisfacción del

- paciente. LUXMÉDICA 5(I5): 23-3I

\section{Abstract}

With the advent and development of new intraocular lenses (IOL's) cataract surgery has evolved into the refractive surgery arena, where new IOL's can satisfactorily replace the natural lens, which represents a major stone in the treatment of presbyopia. This article aims to show up the features of the new IOL's for presbyopia, the clinical results obtained until present and the challenges facing the surgeon, which involves proper selection of patients based on their expectations and needs, selecting the IOL, as well as preoperative evaluation and postoperative care necessary to achieve the maximum level of patient satisfaction.

Palabras clave: Presbicia, catarata, lentes intraoculares.

Keywords: Presbyopia, cataract, intraocular lenses.

* Profesores investigadores de los Departamentos de Medicina y Cirugia del Centro de Ciencias de la Salud, UAA.

** Médico pasante en Servicio Social. Depto. de Cirujía del Centro de Ciencias de la Salud, UAA.

Fecha de recibido: 18-agosto-2010

Fecha de aceptado: 25-agosto-2010

Correspondencia: Torres Bernal LF. Doctor en Ciencias, deptos. de medicina y cirujía, del Centro de Ciencias de la Salud, UAA.

Av. Universidad \#940 ciudad universitaria CP 20100 Ags. Ags. Tel. 910-84-40. Correo electrónico ftorresb_@yahoo.com 


\section{Introducción}

La presbicia es el resultado de una pérdida de la acomodación, es decir, es la incapacidad progresiva de la lente transparente del ojo llamada cristalino, para enfocar objetos cercanos ${ }^{1}$. Existen diversas técnicas que tratan de aliviar este problema y que incluyen la corrección con gafas, la monovisión (tanto con gafas como con cirugía), la ablación multifocal de la córnea con láser excimer, y el implante de nuevos lentes intraoculares (LIO's), ya sean multifocales o acomodativos ${ }^{2}$.

Otra aplicación de los LIO es en la cirugía de catarata, la cual, siempre se ha considerado un procedimiento refractivo. Hasta hace poco, su objetivo se centraba en torno a la eliminación de la catarata sin complicaciones, y la restauración parcial de la visión funcional con el implante de lentes intraoculares. En el pasado, la mayoría de los cirujanos tenían uno o dos "LIO's preferidos" que utilizaban para la mayoría, si no para todos los pacientes. Después de la operación el paciente tenía que usar gafas y / o lentes de contacto para la rehabilitación visual total de lejos y de cerca.

Recientemente, el diseño y fabricación de LIO's ha evolucionado a pasos agigantados, ampliando la posibilidad de imitar la función natural del cristalino humano. La cirugía de catarata con esta nueva tecnología, busca reducir la dependencia de los anteojos y mejorar la calidad de la visión funcional tanto de lejos como de cerca, minimizando los errores de refracción y permitiendo una reincorporación laboral temprana.

\section{Lentes intraoculares para presbicia disponibles.}

Las opciones actualmente aprobadas por la organización conocida como la FDA (por sus siglas en ingles: Food and Drug Admninistration), para la corrección de la presbicia en los E.U.A. incluyen varios lentes multifocales y un lente intraocular acomodativo. Estos lentes multifocales incluyen el más reciente ReZoom (NXG1, Abbott Medical Optics, Abbott Park, IL, E.U.A.) y el ReSTOR (Alcon Laboratories, Fort Worth, Texas, E.U.A.) entre otros. El único lente acomodativo disponible es el Crystalens HD (Bausch \& Lomb, Rochester, N, E.U.A.).

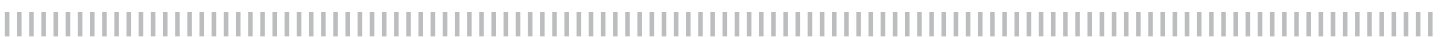

\section{Lentes intraoculares multifocales}

En 2005, la FDA de E.U.A. aprobó dos lentes intraoculares multifocales, uno refractivo, el lente ReZoom (Abbott Medical Optics), y un lente intraocular difractivo, el ReSTOR (Alcon). En 2008, Alcon lanzó el ReSTOR asférico, que proporciona una mejor calidad de visión en comparación con el ReSTOR original ${ }^{3}$.
El LIO AcrySof ReSTOR (Alcon, Fort Worth, Texas, EE.UU.) combina las funciones de dos regiones, una apodizada difractiva y una refractiva. Este es un lente de acrílico hidrofóbico de una sola pieza, con una zona central de $3.6 \mathrm{~mm}$ (dentro de los $6.0 \mathrm{~mm}$. de diámetro óptico total), que contiene 12 escalones concéntricos con alturas que decrecen gradualmente y que distribu- 
yen la energía basados en las condiciones de luminosidad. La región refractiva de la óptica, rodea el área apodizada y se dedica a la visión de lejos. Este lente presenta un poder de adición de 4.0 dioptrías (D) para la visión cercana que se traduce en aproximadamente 3.2 D en el plano de las gafas. Este lente ofrece una excelente agudeza visual sin corrección, con altos índices de independencia de anteojos que se mantienen a largo plazo ${ }^{4,5}$. Los síntomas adversos que pueden presentarse de forma temprana se reducen con el tiempo ${ }^{6}$. En los ensayos clínicos de la FDA, el $80 \%$ de los pacientes con ReSTOR indicaron que nunca usaban gafas para su visión, mientras que el $17 \%$ las usaban algunas veces, y solo el $3 \%$ llevaban gafas todo el tiempo. Aunque la agudeza visual promedio de lejos y cerca son excelentes, se ha reportado que la visión intermedia no es tan buena como con lentes intraoculares monofocales. Sin embargo, en estudios recientes con el nuevo modelo de ReSTOR los pacientes reportaron una visión intermedia funcional? .

El LIO ReZoom (NXG1, Abbott Medical Optics, Abbott Park, IL, E.U.A.) es un lente de acrílico de tres piezas que contiene cinco zonas visuales concéntricas. Este lente se basa en el diseño de zonas progresivas del LIO Array (Advanced Medical Optics, Santa Ana, California, E.U.A.) aprobado por primera vez en 1997. Las dos zonas de cerca dominantes proporcionan poder de adición de cerca de 3.5D en el plano de la LIO y de $2.57 \mathrm{D}$ en el plano de las gafas. El comportamiento óptico del lente, por lo tanto, depende del tamaño de la pupila ${ }^{8}$. Los resultados europeos del lente ReZoom, encontraron que $93.0 \%$ de los pacientes con implante de este LIO nunca o solo ocasionalmente usan gafas ${ }^{9}$. Sin embargo estos pacientes necesitan gafas para lectura o impresión fina. En comparación con otros lentes implantados bilateralmente, los pacientes con ReZoom bilaterales tienen una visión 20/30 o mejor a distancia y un ma- yor número logra la independencia de las gafas, lo que indica que este lente es muy adecuado para corregir la visión a distancia ${ }^{10-12}$. Según los resultados de los ensayos clínicos de la FDA, el 93\% de los pacientes ReZoom fueron independientes de sus gafas para ver a distancia, el $93 \%$ para las tareas intermedias, y el $81 \%$ para la visión cercana.

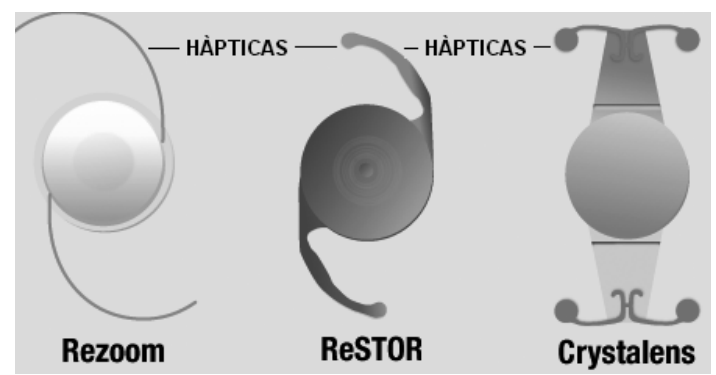

Lentes intraoculares acomodativos

El Crystalens HD (Bausch \& Lomb, Rochester, N, E.U.A.) es el diseño actual del lente acomodativo cuyo mecanismo de acción es controversial. La estructura tenía originalmente una zona óptica de silicón de $4,5 \mathrm{~mm}$ de diámetro con dos hápticas en plato con bisagras flexibles, cada una con dos segmentos de poliamida (modelo AT45). El crystalens se encuentra actualmente aprobado con un diámetro óptico de 5.0 mm (crystalens modelo HD), que utiliza un diseño óptico patentado, optimizado para aumentar la profundidad de foco. El poder de adición de cerca en el plano de las gafas para esta lente es de aproximadamente 1.0$2.5 \mathrm{D}$. Estudios de la FDA han encontrado que $90,1 \%$ de los pacientes presentaron una visión cercana de $\mathrm{J} 3$ o mejor monocularmente (100\% binocular) al año de la cirugía ${ }^{13}$. Estudios del Crystalens indican que este LIO proporciona una mejor agudeza visual sin corrección de cerca y de lejos en comparación con el LIO estándar monofo$\mathrm{cal}^{14}$. En un cuestionario que se distribuyó en un estudio de la FDA en un año después del implante con Crystalens, el 73\% de los pacientes nunca o casi nunca usaban an- 
teojos, el $15 \%$ de ellos los usaba algunas veces y solo el $11 \%$ usaba gafas casi todo el tiempo ${ }^{9}$. En los ensayos de la FDA para una versión anterior del Crystalens (modelo AT-50SE), más del $98 \%$ de los pacientes con implantes bilaterales lograron una agudeza visual de cerca sin corrección de al menos $20 / 40$ (J3) y el $73 \%$ lograron al menos 20/25 (J1). En el ensayo, el 93,8\% de los pacientes con implantes bilaterales, dijo que podían realizar la mayoría de las funciones visuales sin gafas. ${ }^{15}$

\section{Estudios comparativos entre lentes in- traoculares para presbicia}

Existen varios estudios comparativos entre Crystalens ${ }^{13,14}$ ReZoom $^{16}$, y ReSTOR ${ }^{8}$ 17-20 así como estudios de lentes multifocales con controles monofocales. Además se han estudiado en pacientes después de someterse a una cirugía monocular, a cirugía binocular y en varias combinaciones. En estos estudios, los lentes multifocales han demostrado un mejor desempeño de la visión de cerca en los pacientes corregidos con lentes para presbicia que en el grupo de LIO's monofocales. La visión en distancias intermedias es variable, y en general, fue menor con pacientes implantados de forma bilateral con la versión actual de ReSTOR ${ }^{7}$. ${ }^{21-24}$ que con ReZoom o Crystalens, aunque los resultados fueron dependientes de las condiciones de iluminación ${ }^{22,24}$. El Crystalens ha demostrado proporcionar una mejor calidad global de la visión, pero la visión de cerca es menor en comparación con ReSTOR y ReZoom ${ }^{25}$. El ReSTOR ha demostrado proporcionar la mejor visión de lectura sin corrección ${ }^{25}$. Es de destacar que en la mayoría de estos estudios no se han comparado estos lentes con los nuevos lentes monofocales asféricos.

\section{Mezclar y ajustar}

Un pequeño número de cirujanos han iniciado la combinación de las diferentes tecnologías sobre lentes intraoculares para presbicia en un mismo paciente, con el fin de tener los mejores atributos de cada lente y mejorar el resultado visual en su totali- dad. Para algunos cirujanos, esta estrategia pretende reducir al mínimo los inconvenientes de cada modelo de lente y maximizar sus beneficios. Se debe de discutir de forma preoperatoria con el paciente las ventajas de seguir la técnica de mezclar y ajustar, en donde se coloca un ReZoom en el ojo dominante y ReSTOR en el ojo no dominante para maximizar las opciones y satisfacción del paciente ${ }^{7}$.

Algunos cirujanos han encontrado que el crystalens en el ojo dominante y asféricos ReSTOR en el ojo no dominante proporciona al paciente una visión a distancia y de cerca excelente, con una reducción en los fenómenos adversos de visión nocturna ${ }^{25}$. Otros cirujanos recurren a esta técnica de mezcla y ajuste cuando el paciente no está satisfecho con el resultado visual en el primer ojo. En este escenario, el cirujano confía en que mediante el uso de un estilo diferente de lente para presbicia, el rango de visión se amplíe y la satisfacción del paciente se incremente. Un estudio reciente, con la técnica de mezclar y ajustar, colocando ReZoom en el ojo dominante y ReSTOR en el ojo no dominante, demostró una mayor independencia para el uso de gafas y una mayor satisfacción del paciente ${ }^{26}$.

\section{Los efectos secundarios}

Aunque la calidad de vida en general y las respuestas de los pacientes sobre la calidad de visión son prometedoras para estas lentes, existen pacientes aislados (más en los grupos con lentes multifocales) que han informado fenómenos de disfotopsia o disminución en la calidad de visión ${ }^{27}$.

Woodward, et al. analizó y reportó las causas de insatisfacción de los pacientes operados con lentes multifocales dividiéndolas en dos grandes grupos. Aquellas asociadas a visión borrosa que más frecuentemente son producidas por un error refractivo residual y aquellas asociadas a disfotopsia ${ }^{28}$. La disfotopsia pseudofáquica se refiere a un conjunto de síntomas adversos o quejas, que el paciente puede referir posterior al implante de un lente in- 
traocular. Estos síntomas pueden variar en cantidad y calidad, e incluyen deslumbramiento, visión de un halo, rayas luminosas, destellos o ráfagas de luz, o bien una visión nebulosa o sombría, que usualmente se presentan en el campo visual periférico bajo ciertas condiciones de luminosidad. Estos síntomas tienen su origen en un fenómeno óptico dependiente del diseño y material del lente, en donde imágenes indeseables son proyectadas y superimpuestas a la imagen retiniana relevante que se forma directamente por el LIO, causando una degradación del desempeño visual ${ }^{27}$. Esto podría representar un problema bajo ciertas condiciones de iluminación en algunas actividades cotidianas como conducir por la noche o en condiciones de baja visibilidad" ${ }^{1}$. Con los LIO's acomodativos se pueden evitar muchos de estos efectos secundarios, pero también pueden requerir el uso de corrección con gafas para complementar la visión de lectura fina ${ }^{13,29}$.

En una revisión de LIO's multifocales de Cochrane en el 2006 se encontró que los fenómenos de disfotopsia son 3,5 veces más comunes con los LIO's multifocales que con los monofocales ${ }^{30}$. Al aumentar la profundidad de campo 2 a 3 veces más con un LIO multifocal, la sensibilidad al contraste puede disminuir hasta un $50 \%{ }^{31}$, 32. En un estudio realizado por Chiam et al., ${ }^{18} 21.3 \%$ de los pacientes con un LIO ReSTOR reportaron la presencia de un resplandor moderado en comparación con $7,5 \%$ de los pacientes con LIO monofocales. En un estudio realizado por Cillino et al., ${ }^{33}$ los pacientes con LIO's multifocales presentaron una mayor función visual, pero también una mayor incidencia de presencia de halos.

Las causas de los fenómenos de disfotopsia son varias, e incluyen la fibrosis capsular posterior (hasta en un $47 \%$ de los pacientes), el descentramiento del lente, el tamaño de la pupila y las propiedades intrínsecas del lente intraocular ${ }^{28,31,34}$. El cirujano debe de ir descartando las probables causas en cada paciente para que estas quejas puedan ser superadas mediante la neuroadaptación del propio paciente ${ }^{35}$ o bien tratadas de forma eficiente con tratamientos farmacológicos o quirúrgicos $3,34,36$.

Recientemente se ha descrito un efecto colateral del Crystalens conocido como síndrome en Z. Debido a la naturaleza flexible de sus hápticas, la contracción capsular postoperatoria puede producir inestabilidad posicional, y por tanto inestabilidad refractiva de este lente acomodativo. Debido a su diseño en bisagra, el lente es más susceptible que un diseño tradicional a cambios anteriores, posteriores o inclusive a cambios de torsión con la contracción capsular progresiva. Las fuerzas capsulares contráctiles irregulares sobre la bolsa capsular pueden causar torsión anterior de la bisagra, inclinando el óptico y causando este síndrome $Z$, con un marcado cambio astigmático ${ }^{37}$. Se dice que las últimas versiones del lente, el Crystalens 5.0 y los modelos HD, con sus hápticos mayores y más cuadrados, disminuyen la incidencia de esta complicación, aunque todavía puede ocurrir. Se puede requerir tratamiento farmacológico o quirúrgico para su corrección definitiva. Existe un menor número de quejas de los fenómenos de disfotopsia y halos con este lente en comparación con los multifocales ${ }^{25}$.

\section{Desafíos para los nuevos LIO's}

El ser candidato a cirugía facorefractiva implica varios pasos, que inician con la selección adecuada del paciente, en base a su estilo de vida y a sus necesidades visuales. Además de esto, se requiere de un examen ocular preoperatorio completo, así como la educación del paciente respecto a los LIO's refractivos y las opciones quirúrgicas disponibles; el consentimiento informado y las expectativas de los pacientes también deben de ser planteadas, para obtener un nivel mayor de satisfacción postoperatoria.

\section{Selección de los pacientes}

Existen numerosos factores que determinan el éxito de cualquier cirugía de presbi- 
cia con LIO's, pero ninguno es tan importante como la selección del paciente ${ }^{38-42}$.

El reto más difícil para el cirujano es entender las expectativas de cada paciente, para valorar si es posible cumplirlas, con las opciones quirúrgicas disponibles. Para ello se debe valorar la ocupación de cada paciente, conocer sus actividades recreativas, pasatiempos, ubicación geográfica y personalidad. Las preguntas que se realizan incluyen el uso de computadoras, el hábito de lectura, oficios, actividades atléticas y con qué frecuencia conducen de noche. Dell ${ }^{43}$ desarrollo un cuestionario que se aplica a los pacientes y que ayuda a evaluar las necesidades visuales en cuanto al estilo de vida ${ }^{44}$. Se ha observado que los pacientes con alteración en la personalidad como los obsesivos-compulsivos, depresivos y otros tipos de personalidad introspectiva pueden resultar en altos índices de insatisfacción posterior a cualquier tipo de cirugía refractiva ${ }^{9}$.

\section{Evaluación preoperatoria}

El otro reto a vencer es el de intentar predecir el funcionamiento del lente seleccionado en cada paciente. Se debe considerar que los pacientes a los que se les realizará cirugía facorefractiva no deberán de padecer enfermedades oculares adicionales, para garantizar los mejores resultados a futuro.

\section{Astigmatismo corneal}

Es fundamental que el cirujano conozca el astigmatismo preoperatorio del paciente, para poder decidir su manejo adecuado. Los topógrafos Orbscan y Pentacam son útiles para calificar y cuantificar el tipo y grado de astigmatismo, así como para descartar la presencia de queratocono, clínico o frustro, degeneración marginal pelúcida, y otros tipos de astigmatismo irregular ${ }^{45}$. Cuando el paciente presente astigmatismo mayor de $0.75 \mathrm{D}$ se deben utilizar técnicas quirúrgicas correctivas como las incisiones limbares relajantes o queratotomía astigmática que por lo general son eficaces, pero pueden dar resultados inconsistentes en pacientes con más de 2D de astigmatismo. Estos pacientes podrán tratarse con láser excimer en un procedimiento bióptico. Pacientes con astigmatismo irregular no podrán ser candidatos a cirugía facorefractiva de presbicia.

\section{Degeneración Macular}

Las enfermedades maculares, pueden ser contraindicaciones relativas para la cirugía de presbicia con LIO's. La tomografía de coherencia óptica (OCT) de la mácula es útil para detectar la enfermedad en etapa subclínica, que pudieran dar resultados postoperatorios inesperados. La OCT debe realizarse de forma rutinaria en candidatos a colocación de estos LIO's. En pacientes en riesgo de daño macular podemos dar antiinflamatorios no esteroideos (AINE's) una semana anterior a la cirugía y por lo menos cuatro semanas después, utilizando AINE's combinados con esteroides en el período postquirúrgico, ya que estos medicamentos son eficaces en la prevención y tratamiento del edema macular quístico ${ }^{46-48}$.

\section{Antecedentes de cirugías oculares}

Los pacientes con antecedentes de cirugías refractivas y / o de retina pueden ser malos candidatos para cirugía de presbicia intraocular ${ }^{49}$. Esto se da como consecuencia de que estas cirugías influirán en la selección del poder de los LIO's. Tanto en los pacientes con cirugía de retina con aceite de silicón como en los pacientes con cirugía refractiva previa (queratotomía radial, PRK y LASIK) se pueden obtener sorpresas refractivas postoperatorias por la dificultad en la eleccion de LIO's, ${ }^{50}$ debido a un cálculo de lente intraocular deficiente e inexacto ${ }^{51,52}$.

\section{Cuidado postoperatorio}

La disminución en la calidad visual postoperatoria en los pacientes que fueron sometidos a cirugía de presbicia intraocular puede ser resultado de diversas condiciones, como errores refractivos residuales, opacidad de capsular posterior, contracción capsular, disminución de la acomoda- 
ción por la edad o por debilidad zonular, tamaño pupilar inadecuado en diferentes condiciones de iluminación, y/o edema macular quístico. Es pues, necesario considerar y comentar con el paciente antes de la cirugía, que los $\mathrm{LIO}^{\prime}$ s de presbicia por lo general, pueden requerir de un mayor número de procedimientos secundarios que los LIO's monofocales estándar ${ }^{53}$. Estos procedimientos pueden ser capsulotomias YAG (acrónimo del inglés yttrium aluminium garnet), reducción de astigmatismo y errores refractivos, que son necesarios para la satisfacción del paciente.

\section{El consentimiento informado, la infor- mación y manejo de las expectativas del paciente}

El informar adecuadamente al paciente del procedimiento que se le va a realizar, así como el formular el consentimiento informado es crucial, ya que las expectativas de los pacientes preoperatorios desempeñan un papel importante en el estado postoperatorio ${ }^{54}$. En un estudio británico donde se compararon los métodos de educar a los pacientes sobre la cirugía de catarata, se observó que los pacientes que recibieron un vídeo detallado, revisión de la anato- mía de la catarata, así como una explicación de lo que pueden esperar de la cirugía de catarata, obtuvieron menor ansiedad durante el procedimiento y una mayor satisfacción postoperatoria en comparación con los pacientes que sólo recibieron información sobre las consideraciones anatómicas de las cataratas ${ }^{55}$. Otro estudio en Australia concluyó que la manera más efectiva de aumentar la satisfacción del paciente fue por medio de la información dada por los médicos ${ }^{56}$. Es importante recalcar que la mejoría de la función visual en este estudio no se correlacionó con la satisfacción postoperatoria. Los aspectos no médicos de la cirugía de catarata también son importantes para los pacientes; se observó que el tiempo de espera de la cirugía, la ubicación de las citas, la comunicación y comprensión del médico se correlacionaron con una gran satisfacción de los pacientes ${ }^{56}$. La estrategia de prometer menos y dar más, funciona para restablecer la confianza y aceptación de los pacientes. La insatisfacción puede darse por una mala información al paciente, por aberraciones visuales residuales, o expectativas poco realistas.

\section{Conclusión}

La nueva tecnología de LIO's para presbicia y el uso de técnicas quirúrgicas avanzadas han revolucionado la cirugía de catarata, mejorando las expectativas del paciente, logrando la mejoría de su función visual y la satisfacción del mismo. La cirugía refractiva de catarata hace hincapié en que el estilo de vida y las demandas visuales del paciente sean considerados en la selección de procedimientos refractivos y de catarata. En términos generales, si los pacientes desean una buena visión a distancia e intermedia, pero no les importa llevar gafas para trabajar de cerca como la lectura, o manualidades prolongadas pueden ser mejores candidatos para la LIO ReZoom. Los pacientes que deseen una buena visión a distancia y de lectura, pero que no están tan preocupados por la visión intermedia, y que no ocupan hacer mucho trabajo en la computadora, pueden ser mejores candidatos para el lente ReSTOR. Los pacientes que deseen una buena visión a distancia e intermedia, 
pero que pueden estar preocupado por la posibilidad de disfotopsia, y que no les importa esperar un tiempo más largo para alcanzar el beneficio completo de los aspectos acomodativos del cristalino, pueden ser buenos candidatos para el Crystalens. La combinación de los lentes anteriormente descritos en un mismo paciente, puede maximizar sus ventajas y minimizar sus efectos adversos y es una técnica que creemos, será más empleada en el futuro cercano.

\section{Bibliografía}

1 Marchini, G., et al., Anterior segment changes during accommodation in eyes with a monofocal intraocular lens: high-frequency ultrasound study. J Cataract Refract Surg, 2008. 34(6): p. 949-56.

2 Kashani, S., A.A. Mearza, and C. Claoue, Refractive lens exchange for presbyopia. Cont Lens Anterior Eye, 2008. 31(3): p. 117-21.

3 Choi, J. and J. Schwiegerling, Optical performance measurement and night driving simulation of ReSTOR, ReZoom, and Tecnis multifocal intraocular lenses in a model eye. J Refract Surg, 2008. 24(3): p. 218-22.

4 Belani S, M.S., ReSTOR in patients 80 years of age and older., in American Society of Cataract and Refractive Surgery, A.S.o.C.a.R. Surgery, Editor. 2008: Chicago, Illinois.

5 Fisher, B., Experience with an apodized diffractive aspheric IOL., in American Society of Cataract and Refractive Surgery, A.S.o.C.a.R. Surgery, Editor. 2008: Chicago, Illinois.

6 Kaufer, R., Outcomes 3 years after bilateral implantation of an apodized diffractive IOL., in American Society of Cataract and Refractive Surgery, A.S.o.C.a.R. Surgery, Editor. 2008: Chicago, Illinois.

7 Blaylock, J.F., Z. Si, and C. Vickers, Visual and refractive status at different focal distances after implantation of the ReSTOR multifocal intraocular lens. J Cataract Refract Surg, 2006. 32(9): p. 1464-73.

8 Ortiz, D., et al., Optical performance of monofocal and multifocal intraocular lenses in the human eye. J Cataract Refract Surg, 2008. 34(5): p. 755-62.

9 Packer M, F.I., Hoffman RS, Refractive lens exchange, in Focal Points. 2007, Focal Points. p. 8.

10 McCabe, C., Comparing visual results and patient satisfaction with bilateral implantation of ReZoom and ReSTOR IOLs., in American Society of Cataract and Refractive Surgery, A.S.o.C.a.R. Surgery, Editor. 2008: Chicago, Illinois.

11 Parekh, P., Outcomes of presbyopic intraocular lenses., in American Society of Cataract and Refractive
Surgery, A.S.o.C.a.R. Surgery, Editor. 2008: Chicago, Illinois.

12 Rau, M., Mix and match versus bilateral implantation of ReZoom and bilateral implantation of Tecnis multifocal IOLs., in American Society of Cataract and Refractive Surgery, A.S.o.C.a.R. Surgery, Editor. 2008 : Chicago, Illinois.

13 Cumming, J.S., et al., Clinical evaluation of the Crystalens AT-45 accommodating intraocular lens: results of the U.S. Food and Drug Administration clinical trial. J Cataract Refract Surg, 2006. 32(5): p. 81225

14 Macsai, M.S., L. Padnick-Silver, and B.M. Fontes, Visual outcomes after accommodating intraocular lens implantation. J Cataract Refract Surg, 2006. 32(4): p. 628-33.

15 Santaella, R. and N. Afshari, Presbyopia-correcting intraocular lenses: 'one lens does not fit all'. Curr Opin Ophthalmol, 2010. 21(1): p. 1-3.

16 Martinez Palmer, A., et al., Visual function with bilateral implantation of monofocal and multifocal intraocular lenses: a prospective, randomized, controlled clinical trial. J Refract Surg, 2008. 24(3): p 257-64.

17 Rocha, K.M., et al., Postoperative wavefront analysis and contrast sensitivity of a multifocal apodized diffractive IOL (ReSTOR) and three monofocal IOLs. J Refract Surg, 2005. 21(6): p. S808-12.

18 Chiam, P.J., et al., ReSTOR intraocular lens implantation in cataract surgery: quality of vision. J Cataract Refract Surg, 2006. 32(9): p. 1459-63.

19 Souza, C.E., et al., Visual performance of AcrySof ReSTOR apodized diffractive IOL: a prospective comparative trial. Am J Ophthalmol, 2006. 141(5): p. 827-832.

20 Vingolo, E.M., et al., Visual acuity and contrast sensitivity: AcrySof ReSTOR apodized diffractive versus AcrySof SA60AT monofocal intraocular lenses. J Cataract Refract Surg, 2007. 33(7): p. 1244-7.

21 Alfonso, J.F., et al., Prospective visual evaluation of apodized diffractive intraocular lenses. J Cataract Refract Surg, 2007. 33(7): p. 1235-43. 
22 Alfonso, J.F., et al., Correlation of pupil size with visual acuity and contrast sensitivity after implantation of an apodized diffractive intraocular lens. J Cataract Refract Surg, 2007. 33(3): p. 430-8.

23 Chiam, P.J., et al., Functional vision with bilateral ReZoom and ReSTOR intraocular lenses 6 months after cataract surgery. J Cataract Refract Surg, 2007. 33(12): p. 2057-61.

24 Hutz, W.W., et al., Intermediate vision and reading speed with array, Tecnis, and ReSTOR intraocular lenses. J Refract Surg, 2008. 24(3): p. 251-6.

25 Pepose, J.S., et al., Visual performance of patients with bilateral vs combination Crystalens, ReZoom, and ReSTOR intraocular lens implants. Am J Ophthalmol, 2007. 144(3): p. 347-357.

26 Akaishi L, F.P., PC IOLs mix and match technologies: Brazilian experience., in World Ophthalmology Congress. 2006.: Sao Paolo, Brazil.

27 Schwiegerling, J., Recent developments in pseudophakic dysphotopsia. Curr Opin Ophthalmol, 2006. 17(1): p. 27-30.

28 Woodward, M.A., J.B. Randleman, and R.D. Stulting, Dissatisfaction after multifocal intraocular lens implantation. J Cataract Refract Surg, 2009. 35(6): p. 992-7.

29 Olson, R.J., Presbyopia correcting intraocular lenses: what do I do? Am J Ophthalmol, 2008. 145(4): p. 593-4.

30 Leyland, M., Pringle E. Multifocal versus monofocal intraocular lenses after cataract extraction. 2006 [cited 2009; Available from: www.cochrane.org.

31 Hayashi, K., et al., Correlation between pupillary size and intraocular lens decentration and visual acuity of a zonal-progressive multifocal lens and a monofocal lens. Ophthalmology, 2001. 108(11): p. 2011-7.

32 Pieh, S., et al., Halo size under distance and near conditions in refractive multifocal intraocular lenses. $\mathrm{Br} \mathrm{J}$ Ophthalmol, 2001. 85(7): p. 816-21.

33 Cillino, S., et al., One-year outcomes with new-generation multifocal intraocular lenses. Ophthalmology, 2008. 115(9): p. 1508-16.

34 Baumeister, M., et al., Tilt and decentration of threepiece foldable high-refractive silicone and hydrophobic acrylic intraocular lenses with 6-mm optics in an intraindividual comparison. Am J Ophthalmol, 2005. 140(6): p. 1051-8.

35 Pepose, J.S., Maximizing satisfaction with presbyopia-correcting intraocular lenses: the missing links. Am J Ophthalmol, 2008. 146(5): p. 641-8.

36 Artigas, J.M., et al., Image quality with multifocal intraocular lenses and the effect of pupil size: comparison of refractive and hybrid refractive-diffractive designs. J Cataract Refract Surg, 2007. 33(12): p. 2111-7.

37 Cazal, J.M., Cosme Lavin-Dapena, Jorge MD, and J.O. Marín, and Vergés Carlos Accommodative Intraocular Lens Tilting. AMERICAN JOURNAL OF OPHTHALMOLOGY, 2005. 140: p. 4.

38 Gray, P.J. and M.G. Lyall, Diffractive multifocal intraocular lens implants for unilateral cataracts in pre- presbyopic patients. Br J Ophthalmol, 1992. 76(6): p. 336-7.

39 Findl, O., Intraocular lenses for restoring accommodation: hope and reality. J Refract Surg, 2005. 21(4): p. 321-3.

40 Doane, J.F. and R.T. Jackson, Accommodative intraocular lenses: considerations on use, function and design. Curr Opin Ophthalmol, 2007. 18(4): p. 31824.

41 Kohnen, T., et al., [Use of multifocal intraocular lenses and criteria for patient selection]. Ophthalmologe, 2008. 105(6): p. 527-32.

42 Pepin, S.M., Neuroadaptation of presbyopia-correcting intraocular lenses. Curr Opin Ophthalmol, 2008. 19(1): p. 10-2.

43 Dell, S., Screening and evaluating presbyopic patients., in Cataract Refract Surg Today. 2007, Cataract Refract Surg Today. p. 2

44 Dell, S. Cataract and refractive lens exchange questionnaire. 2008 Accessed 18 August 2008]; Available from: http://www.crstoday.com/Pages/patientQ.php.

45 Trattler W, B.C., Pearls for success with presbyopia-correcting IOLs, in Cataract Refract Surg Today. 2007, Cataract Refract Surg Today. p. 2.

46 Samiy, N. and C.S. Foster, The role of nonsteroidal antiinflammatory drugs in ocular inflammation. Int Ophthalmol Clin, 1996. 36(1): p. 195-206.

47 Rho, D.S., Treatment of acute pseudophakic cystoid macular edema: Diclofenac versus ketorolac. J Cataract Refract Surg, 2003. 29(12): p. 2378-84.

48 O'Brien, T.P., Emerging guidelines for use of NSAID therapy to optimize cataract surgery patient care. Curr Med Res Opin, 2005. 21(7): p. 1131-7.

49 Devgan, U., Cataract surgery after radial keratotomy can be challenging, in Ocular Surg News. 2007, Ocular Surg News.

50 Packer, M., et al., Intraocular lens power calculation after incisional and thermal keratorefractive surgery. J Cataract Refract Surg, 2004. 30(7): p. 1430-4.

51 Hoffer, K.J., Ultrasound velocities for axial eye length measurement. J Cataract Refract Surg, 1994. 20(5): p. 554-62.

52 Byrne SF, G.R., Axial length measurements (A-scan biometry). in Ultrasound of the eye and orbit, 2nd ed., G.R. Byrne SF, editors., Editor. 2002, Mosby: St Louis, Missour. p. 35-37.

53 Leccisotti, A., Secondary procedures after presbyopic lens exchange. J Cataract Refract Surg, 2004. 30(7): p. 1461-5.

54 Nijkamp, M.D., et al., Determinants of patient satisfaction after cataract surgery in 3 settings. J Cataract Refract Surg, 2000. 26(9): p. 1379-88.

55 Pager, C.K., Randomised controlled trial of preoperative information to improve satisfaction with cataract surgery. Br J Ophthalmol, 2005. 89(1): p. 10-3.

56 Pager, C.K., Assessment of visual satisfaction and function after cataract surgery. J Cataract Refract Surg, 2004. 30(12): p. 2510-6. 